\title{
Replicating the success of mitral valve repair in the aortic valve
}

\author{
Joel Price, MD, MPH, FRCSC
}

From the Division of Cardiac Surgery, University of British Columbia, Vancouver, British Columbia, Canada. Disclosures: Author has nothing to disclose with regard to commercial support.

Received for publication July 22, 2018; accepted for publication July 22, 2018; available ahead of print Aug 31, 2018.

Address for reprints: Joel Price, MD, MPH, FRCSC, Division of Cardiac Surgery, University of British Columbia, 494-1081 Burrard St, Vancouver, BC V6Z 1Y6, Canada (E-mail: joel.price@vgh.ca).

J Thorac Cardiovasc Surg 2019;157:124-5

$0022-5223 / \$ 36.00$

Copyright (c) 2018 by The American Association for Thoracic Surgery

https://doi.org/10.1016/j.jtcvs.2018.07.070

Mitral valve repair has been fabulously successful, not only in terms of improving outcomes for treatment of degenerative mitral valve regurgitation but also in the degree to which these techniques have been adopted on a wide scale. ${ }^{1}$ Most centers practicing cardiac surgery today will have one or many surgeons specializing in mitral valve reconstruction. One of the reasons for this success is a reliable method to evaluate the likelihood of a successful repair intraoperatively, before the crossclamp is removed. This is frequently done by the instillation of saline solution to pressurize the left ventricle and visualize any significant residual leak. Aortic valve repair (AVr) is in its relative infancy compared with mitral repair. In general, uptake of this procedure has been limited, despite numerous publications demonstrating positive results. One important obstacle is the absence of a reliable and objective intraoperative assessment similar to that described for the mitral valve. It is generally not feasible to pressurize the open aortic root. If the root is pressurized, it cannot be simultaneously visualized.

In their article in this issue of the Journal, Berra and colleagues $^{2}$ describe a device that they have developed to address this challenge. The study tested the device on explanted porcine hearts, in which the aortic valves were subjected to various AVr techniques. The device was then affixed to the ascending aorta, and the aortic root was pressurized with crystalloid solution. The valve was then visualized with an endoscope, and either a plastic ruler or metal caliper was introduced to measure coaptation length, a value that has been demonstrated to be a predictor of successful aortic repair when measured echocardiographcially. ${ }^{3}$

This device represents a potentially elegant method for intraoperative testing of AVr. There are, however, a number of challenges to extrapolating its use from an explanted porcine heart to the clinical situation. As described, it is required to leave $5 \mathrm{~cm}$ of ascending aorta above the sinotubular junction to affix the device high enough to avoid distorting the aortic valve. This is problematic, because most surgeons perform the aortotomy significantly lower to

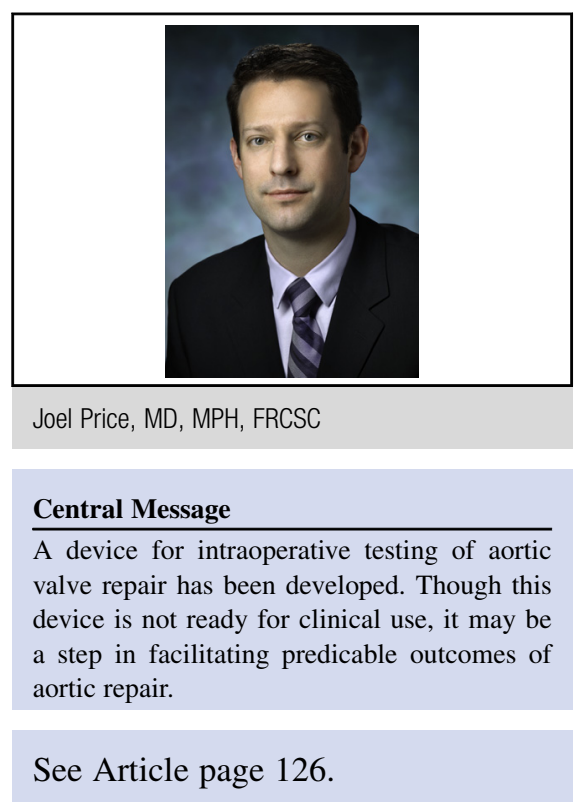

facilitate the repair. Many surgeons, however, perform AVr with an aortic valve-sparing root replacement procedure as the annuloplasty. Although Berra and colleagues ${ }^{2}$ have not yet examined this, I suspect that the device would work very well if attached to the top of the Dacron polyester


of the coronary arteries to achieve pressurization of the aortic root. Doing this in the clinical setting would be unpalatable. Even occluding the coronaries internally, as suggested by Berra and colleagues, ${ }^{2}$ would add a degree of risk to the procedure. This issue will have to be addressed further if the device is to be clinically successful. It is my belief that occluding the coronaries is not necessary to perform the measurements. To mimic the natural physiologic state of the root more closely, continuous infusion of a crystalloid solution could be used while performing inspection and measurements.

To be clear, this device as described is not nearly ready for clinical use. There are a number of challenges that need to be addressed. Berra and colleagues, ${ }^{2}$ however, recognize the importance of this critical step in facilitating predicable outcomes and increased uptake of this useful set of techniques. Ultimately, improved intraoperative assessment will allow increasing numbers of patients to benefit from $\mathrm{AVr}$, and the efforts of Berra and colleagues ${ }^{2}$ are laudable. 


\section{References}

1. Carpentier A, Adams DH, Filsoufi F. Carpentier's Reconstructive Valve Surgery: From Valve Analysis to Valve Reconstruction. Maryland Heights (MO): Saunders; 2010.

2. Berra IG, Hammer PE, Berra S, Irusta AO, Ryu SC, Perrin DP, et al. An intraoperative test device for aortic valve repair. J Thorac Cardiovasc Surg. 2019;157:126-32.

3. le Polain de Waroux JB, Pouleur AC, Robert A, Pasquet A, Gerber BL, Noirhomme P, et al. Mechanisms of recurrent aortic regurgitation after aortic valve repair: predictive value of intraoperative transesophageal echocardiography. JACC CardiovasC Imaging. 2009;2: 931-9. 